\title{
WHISPers, the USGS-NWHC Wildlife Health Information Sharing Partnership Event Reporting System
}

\author{
Julianna B. Lenoch* \\ National Wildlife Health Center, Madison, WI, USA
}

\section{Objective}

The USGS National Wildlife Health Center in conjunction with federal, state, tribal partners proposed an event reporting system with current and historic information on wildlife morbidity and mortality events in North America. The vehicle to accomplish this goal is WHISPers, the Wildlife Health Information Sharing Partnership event reporting system. This system depicts laboratory confirmed wildlife mortality events using geospatial mapping capability. Data are collected by multiple partners to collectively enhance the understanding of disease in wildlife populations.

\section{Introduction}

Although national surveillance systems are maintained for human health (CDC) and for livestock disease (USDA); there is no network or data repository in the area of wildlife disease surveillance. Because emerging and re-emerging diseases severely affect wildlife populations, impact domestic and agricultural animals, and are a reservoir for zoonotic transmission, it is crucial to have early notification and recognition of disease patterns in wildlife populations. Due to fragmented systems of wildlife management, inconsistent investigation into sudden mortality events, and limited laboratory availability, there is not a single entity that is responsible for reporting disease events in North American wildlife populations.

\section{Methods}

In effort to create a readily available data repository for wildlife disease events, NWHC created a partner-driven online database for recording on-going and historical wildlife morbidity and mortality events. Although the system was initially populated with NWHC's $30+$ year database on US wildlife morbidity and mortality events, a portal for data entry by other wildlife professionals is currently underway. The software system continuously updates reported events. The database can be used to improve recognition of seasonally or cyclically epidemic diseases. Many diseases are quite predictable, occurring in the same locations at similar times each year. By chronicling these "typical" events, we can develop a better picture of disease impacts on wildlife across North America. Knowledge of "typical" events also make it easier to identify new diseases as they emerge and potentially spread across the landscape. A centralized repository of this information promotes better awareness of wildlife disease and augments opportunity for both proactive and timely reactive response by natural resource managers.

\section{Results}

The data informatics team at the USGS National Wildlife Health Center created WHISPers to meet the National need for a singular reporting system for wildlife disease events. Confirmed events that meet these guidelines:

1) 5 or more animals with illness or death in a defined geographic area

2) Laboratory confirmed cases

3) Reportable at the county level to protect sensitive information and landowner privacy
4) Diagnosis is categorically attributed to infectious origin (viral, bacterial, fungal, parasitic), traumatic, nutritional, toxic, or other

Sick or dead animals must be observed, collected, submitted to a diagnostic laboratory, and the information shared in WHISPers in order to appear on the map and in the associated database. WHISPers may be used to report morbidity events, mortality events from infectious disease (like avian cholera) and sudden die-offs following environmental toxicity (Harmful Algal Blooms).

The collected data in WHISPers is available publically and free of charge. This information may be used by wildlife scientists, researchers, and natural resource managers. The data may also serve to alert of events that may affect agricultural animal or human populations, supportive the concept of OneHealth surveillance.

\section{Conclusions}

Technological challenges prevent wildlife agencies, diagnostic laboratories, and other organizations from sharing knowledge of mortality events. The introduction of WHISPers should improve the access to data for wildlife mortality events in the United States. A long-term goal for WHISPers is to grow the numbers of contributing partners and make it easier for data to be shared.

Improved usage and submission to WHISPers by partners at the federal, state, tribal and local level will help improve accuracy and completeness of the surveillance system.

\section{Keywords}

Wildlife; Surveillance; Management; Detection; Zoonotic

\section{Acknowledgments}

The concept for WHISPers was developed by the USGS National Wildlife Health Center, in partnership with federal, state, tribal, non-governmental, and academic partners. System software was developed by the USGS National Wildlife Health Center.

\section{References}

https://www.nwhc.usgs.gov/whispers/

*Julianna B. Lenoch

E-mail: julielenoch@gmail.com 